EPJ manuscript No.

(will be inserted by the editor)

\title{
Two-Pion Production in Proton-Proton Collisions with Polarized Beam
}

S. Abd El-Bary ${ }^{8}$, S. Abd El-Samad ${ }^{8}$, R. Bilger ${ }^{6}$, K.-Th. Brinkmann ${ }^{10}$, H. Clement ${ }^{6}$, M. Dietrich ${ }^{6}$, E. Doroshkevich ${ }^{6}$, S. Dshemuchadse ${ }^{5,2}$, A. Erhardt ${ }^{6}$, W. Eyrich ${ }^{3}$, A. Filippi ${ }^{7}$, H. Freiesleben ${ }^{2}$, M. Fritsch ${ }^{3,1}$, R. Geyer ${ }^{4}$, A. Gillitzer ${ }^{4}$,

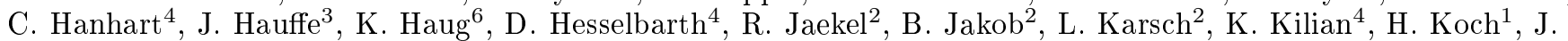
$\mathrm{Kress}^{6}$, E. Kuhlmann ${ }^{2}$, S. Marcello ${ }^{7}$, S. Marwinski ${ }^{4}$, R. Meier ${ }^{6}$, K. Möller ${ }^{5}$, H.P. Morsch ${ }^{4}$, L. Naumann ${ }^{5}$, J. Ritman ${ }^{4}$, E. Roderburg ${ }^{4}$, P. Schönmeier ${ }^{2,3}$, M. Schulte-Wissermann ${ }^{2}$, W. Schroeder ${ }^{3}$, M. Steinke ${ }^{1}$, F. Stinzing ${ }^{3}$, G.Y. Sun ${ }^{2}$, J. Wächter ${ }^{3}$, G.J. Wagner ${ }^{6}$, M. Wagner ${ }^{3}$, U. Weidlich ${ }^{6}$, A. Wilms ${ }^{1}$, P. Wintz ${ }^{8}$, S. Wirth ${ }^{3}$, G. Zhang ${ }^{6 a}$, and P. Zupranski $^{9}$

1 Ruhr-Universität Bochum, Germany

2 Technische Universität Dresden, Germany

${ }^{3}$ Friedrich-Alexander-Universität Erlangen-Nürnberg, Germany

${ }^{4}$ Forschungszentrum Jülich, Germany

${ }^{5}$ Forschungszentrum Rossendorf, Germany

${ }^{6}$ Physikalisches Institut der Universität Tübingen, Auf der Morgenstelle 14, D-72076 Tübingen, Germany

${ }^{7}$ University of Torino and INFN, Sezione di Torino, Italy

8 Atomic Energy Authority NRC Cairo, Egypt

${ }^{9}$ Soltan Institute for Nuclear Studies, Warsaw, Poland

10 Rheinische Friedrich-Wilhelms Universität Bonn, Germany

(COSY-TOF Collaboration)

June 24, 2008

\begin{abstract}
The two-pion production reaction $p p \rightarrow p p \pi^{+} \pi^{-}$was measured with a polarized proton beam at $T_{p} \approx 750$ and $800 \mathrm{MeV}$ using the short version of the COSY-TOF spectrometer. The implementation of a delayed pulse technique for Quirl and central calorimeter provided positive $\pi^{+}$identification in addition to the standard particle identification, energy determination as well as time-of-flight and angle measurements. Thus all four-momenta of the emerging particles could be determined with 1-4 overconstraints. Total and differential cross sections as well as angular distributions of the vector analyzing power have been obtained. They are compared to previous data and theoretical calculations. In contrast to predictions we find significant analyzing power values up to $A_{y}=0.3$.
\end{abstract}

PACS. 13.75.Cs $-25.10 .+\mathrm{s}-25.40 . \mathrm{Ep}-29.20 . \mathrm{Dh}$

\section{Introduction}

The $\Delta(1232)$ resonance is the most dominant resonance in the pion-nucleon system. Therefore pion production in nucleon-nucleon collisions is strongly affected by its excitation if kinematically and dynamically allowed [1,2]. This is not the case in two-pion production near threshold. Since the $\Delta$ resonance decays by emission of only one pion, single- $\Delta$ excitation can contribute to two-pion production only if a second pion is produced associatedly. However, near the two-pion production threshold it is very unlikely that the second nucleon involved in the collision process gets excited, too. Hence the associated production of the second pion can only proceed by non-resonant rescatter-

\footnotetext{
${ }^{a}$ present address: Peking University

Correspondence to: $\mathrm{H}$. Clement

email: clement@pit.physik.uni-tuebingen.de
}

ing, which however, is a very weak process as calculated by Ref. [3].

This situation provides a unique opportunity to study excitation and decay of the Roper resonance $N^{*}(1440)$, because this process provides the only possibility for resonant two-pion production at energies close to the two-pion threshold - as has been predicted in detailed theoretical calculations 3 and as has been demonstrated by the first exclusive measurements performed by the PROMICE/ WASA collaboration at the CELSIUS ring in the energy range of $T_{p}=650-775 \mathrm{MeV}$ [4,5,6].

The measured angular distributions in the center-ofmass system (cms) are all consistent with isotropy with the exception of the proton angular distribution, which is shaped by the dominating $\sigma$ exchange between the two colliding protons - as demonstrated in Ref. [4] and predicted by Ref. [3]. From this we learn that except for the 


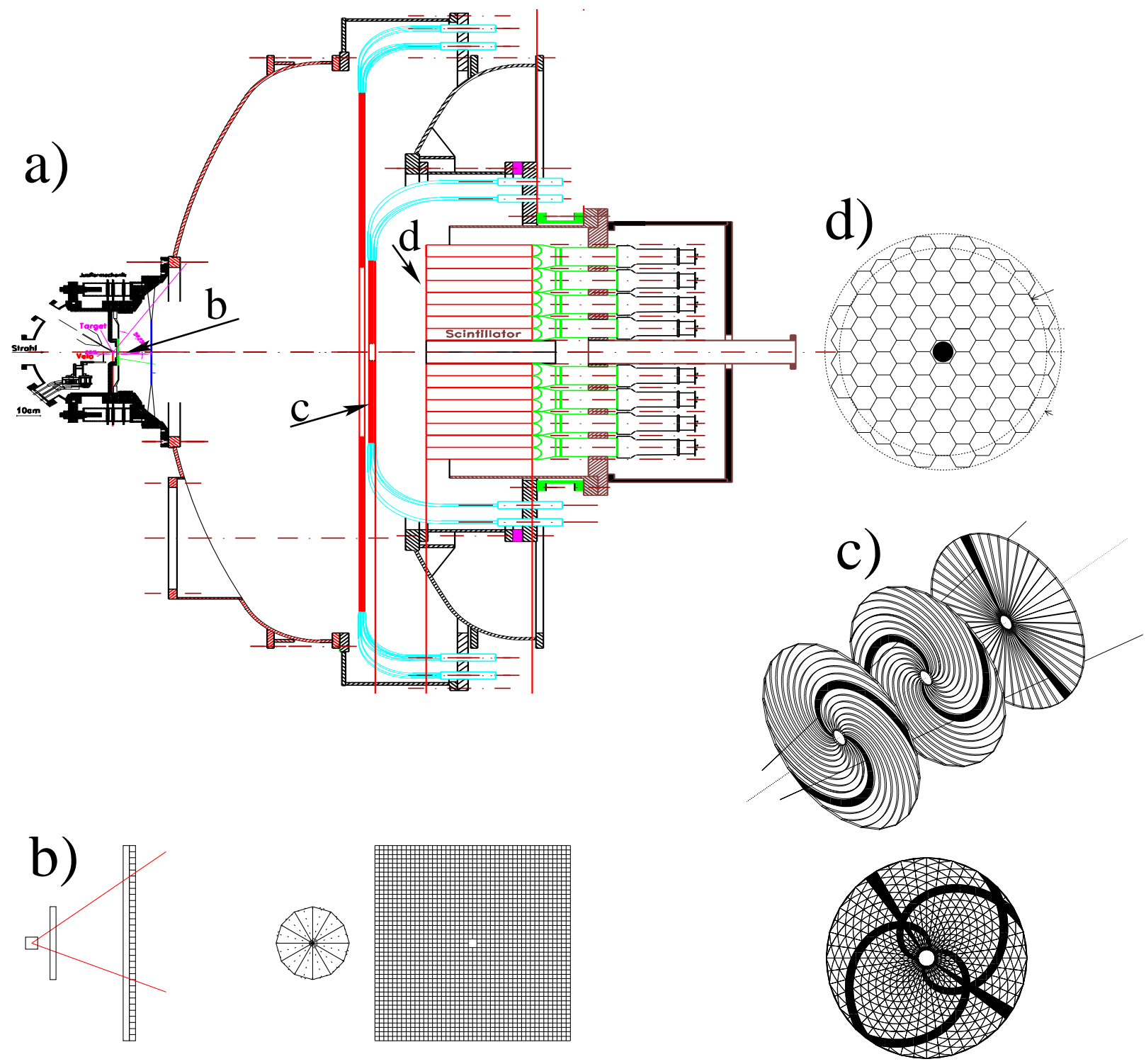

Fig. 1. Sketch of the short COSY-TOF setup used in this experiment showing (a) the full detector arrangement with inserts for (b) the start detector region with start wedges and hodoscope displaying both side (left) and front (right) views, (c) the central hodoscope ("Quirl") as the stop detector for TOF measurements and (d) the central calorimeter. The positions of the subdetector systems (b) - (d) in the full setup are indicated in (a). For a detailed description see text. 
relative motion of the two protons all ejectiles are in relative s-waves to each other at beam energies close to threshold. This is not surprising, since the leading mechanism for two-pion production, which is the excitation of the Roper resonance and its subsequent decay $N^{*} \rightarrow N \sigma$ into the $\sigma$ channel $(\pi \pi)_{I=l=0}$, produces only particles in relative s-waves. Also the Roper decay via the $\Delta$ resonance $N^{*} \rightarrow \Delta \pi \rightarrow N \pi^{+} \pi^{-}$produces predominantly only relative s-waves, although both pions are produced in a pwave. Since no evidence of any significant p-wave contribution (except partially between the two emitted protons) is observed in the differential cross section, the dominating partial wave for two-pion production near threshold must be the ${ }^{1} S_{0}$ partial wave in the entrance channel. However, for non-zero analyzing powers at least two interfering partial waves are necessary. Hence, from the Roper excitation and decay we do not expect sizable non-zero analyzing power values. Indeed, the calculations of Ref. [3], which include also the two-pion production via the $\Delta \Delta$ system, predict vanishing analyzing powers. This result, however, should be taken with some caution, since the calculations have been carried out without taking into account initial state interactions, which should be a prime source of imaginary parts of the amplitudes and analyzing powers are proportional to the imaginary part of interference terms.

On the other hand analyzing powers originate from the interference of amplitudes. Hence small amplitudes in the production process, in particular p-wave amplitudes, can be easily sensed in polarized beam measurements

\section{Experiment}

The measurements have been carried out at the Jülich Cooler Synchrotron COSY at beam energies of nominally 747 and $793 \mathrm{MeV}$ using the time-of-flight spectrometer TOF at an external beam line. The setup of the TOF detector system is displayed in Fig. 1. At the entrance of the detector system the beam - focussed to a diameter smaller than $2 \mathrm{~mm}$ - hits the $\mathrm{LH}_{2}$ target, which has a length of $4 \mathrm{~mm}$, a diameter of $6 \mathrm{~mm}$ and $0.9 \mu \mathrm{m}$ thick hostaphan foils as entrance and exit windows [7]. At a distance of $22 \mathrm{~mm}$ downstream of the target the two layers of the start detector (each consisting of $1 \mathrm{~mm}$ thick scintillators cut into 12 wedge-shaped sectors) were placed followed by a two-plane fiber hodoscope (96 fibers per plane, $2 \mathrm{~mm}$ thick fibers ) at a distance of $165 \mathrm{~mm}$ from target, see Fig. 1b. Whereas the start detector mainly supplies the start times for the time-of-flight (TOF) measurements, the fiber hodoscope primarily provides a good angular resolution for the detected particle tracks. In its central part the TOF-stop detector system consists of the so-called Quirl, a 3-layer scintillator system $1081 \mathrm{~mm}$ downstream of the target shown in Fig. 1c and described in detail in Ref. [8] - and in its peripheral part of the so-called Ring, also a 3layer scintillator system built in a design analogous to the Quirl, however, with inner and outer radii of 560 and 1540 $\mathrm{mm}$, respectively. Finally behind the Quirl a calorimeter (Fig. 1a,d) was installed for identification of charged particles and of neutrons as well as for measuring the energy
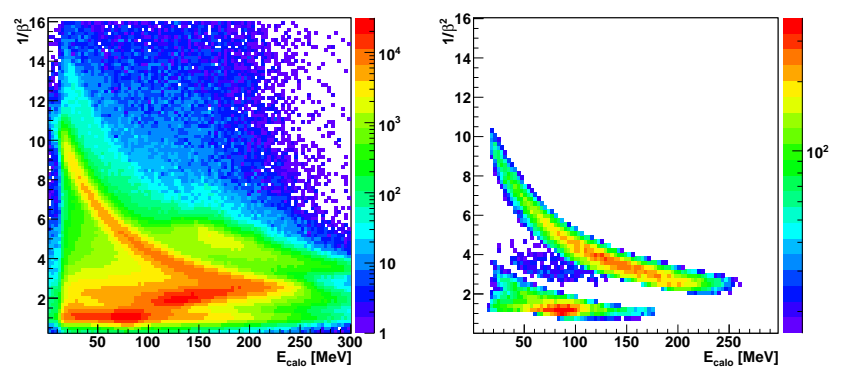

Fig. 2. $\Delta E-E$ plots for particles hitting the calorimeter. The $\Delta E$ information is taken from the TOF information obtained by Quirl and start detector and plotted as $1 / \beta^{2}$, where $\beta=v / c$ denotes the particle velocity relative to c. For the $E$ information the energy deposited in the calorimeter has been taken. Left: without any trigger selections and conditions. Right: with trigger selection for three- and four-prong events as well as with missing mass conditions $M M_{p p} \geq 260 \mathrm{MeV}$ and $100 \mathrm{MeV}$ $\leq M M_{p p \pi} \leq 180 \mathrm{MeV}$, see text.

of charged particles. The calorimeter, details of which are given in Ref. [9], consists of 84 hexagon-shaped scintillator blocks of length $450 \mathrm{~mm}$, which suffices to stop deuterons, protons and pions of energies up to 400,300 and $160 \mathrm{MeV}$, respectively.

The particle identification is done with the standard $\Delta \mathrm{E}-\mathrm{E}$ technique using the energy signal from the calorimeter together with the TOF-signal of the Quirl as shown in Fig. 2 and as described in more detail in Ref.[10], where also sample spectra are shown.

In order to distinguish $\pi^{+}$and $\pi^{-}$particles we have implemented the delayed-pulse technique into the calorimeter setup. By using multihit TDCs the decay of a $\mu^{+}$ resulting from the decay of a $\pi^{+}$stopped in one of the calorimeter blocks can be registered by an afterpulse arriving in the $\mu \mathrm{s}$ range after the prompt pulse. In order to prevent false afterpulses to be registered, which stem from prompt pulses arising from follow-up events, we use the last layer of the Quirl as veto for afterpulses. This technique for $\pi^{+}$identification had previously been installed at the forward detector of PROMICE/WASA and CELSIUS/WASA, respectively, where it has been shown to work reliably [11,12]. Fig. 3 shows a sample spectrum of registered delayed pulses at a proton beam of $T_{p}=793$ $\mathrm{MeV}$. The spectrum is very clean following the expected exponential decay pattern of $\mu^{+}$in matter with a lifetime of $2.2 \mu \mathrm{s}$. The overall efficiency of this $\pi^{+}$identification has been determined to be $39 \%$. The efficiency losses mainly stem from up-stream moving positrons, which originate from $\mu^{+}$decay in the calorimeter and create a veto signal in the last layer of the Quirl.

In the experiment the trigger for two-pion production was set to at least three hits in the Quirl-Ring system associated with two hits in the start detector. That way single-pion production events could be excluded, which at most can give two hits in the Quirl-Ring system. For calibration and monitoring purposes, however, a two-prong trigger was also set up with a high prescale factor. From 
straight-line fits to the hit detector elements the tracks of charged particles were reconstructed. They are accepted as good tracks, if they originate in the target and have a hit in each detector element the track passes through. In this way the angular resolution is better than $1^{\circ}$ both in azimuthal and in polar angles. By construction of the calorimeter a particle may hit one or more calorimeter blocks. The number of blocks hit by a particular particle is given by the track reconstruction. The total energy deposited by this particle in the calorimeter is then just the (calibrated) sum of energies deposited in all blocks belonging to the particular track.

In order to have maximum angular coverage by the detector elements and to minimize the fraction of charged pions decaying in flight before reaching the stop detectors, the short version of the TOF spectrometer was used. In this way a total polar angle coverage of $3^{\circ} \leq \Theta^{l a b} \leq$ $49^{\circ}$ was achieved with the central calorimeter covering the region $3^{\circ} \leq \Theta^{l a b} \leq 28^{\circ}$.

By identifying and reconstructing three or four charged tracks of an event the reaction channel $p p \pi^{+} \pi^{-}$can be fully identified and reconstructed kinematically complete with 1 - 4 overconstraints. The maximum possible laboratory (lab) polar angle for protons emerging from twopion production events is $\approx 30^{\circ}$. Hence practically all protons are within the angular acceptance of the calorimeter and can be identified as protons there. For charged pions emerging from two-pion production events there is no kinematic limit at the incident beam energies of interest. Hence the angular coverage has not been complete for their detection with this setup. However, since detection of two protons and one pion are enough to safely reconstruct the second pion of an event and since due to the identity of the initial collision partners the unpolarized angular distributions are symmetric about $90^{\circ}$ in the overall center-of-mass system (cms), still most of the reaction phase space has been covered by this experiment. The total acceptance has been $15 \%$ of the full phase space, which is a factor $5-7$ larger than in the PROMICE/WASA measurements, where the angular acceptance was $\leq 21^{\circ}$.

Though most of the events stemming from single-pion production could be removed by the three- and four-prong trigger conditions, there is still appreciable background left from $p p \pi^{0}$ events, where the $\pi^{0}$ either undergoes Dalitz decay or where the $\gamma_{\mathrm{s}}$ emerging from $\pi^{0}$ decay undergo conversion in matter. In both cases $e^{+} e^{-}$pairs are produced, which easily may be misidentified as charged pions. And since the $p p \pi^{0}$ production cross section is three orders of magnitude larger than that for $p p \pi^{+} \pi^{-}$this background is sizeable. In order to suppress this background we applied two constraints on missing mass spectra as shown in Fig.4. For the missing mass $M M_{p p \pi}$ of two identified protons and one identified pion, which has to be equivalent to the mass of the second pion in case of a true twopion production event, we require $100 \mathrm{MeV} \leq M M_{p p \pi} \leq$ $180 \mathrm{MeV}$. We see from Fig. 4 that by this condition the $M M_{p p}$ spectrum gets already very clean. To be on the safe side we introduce as further constraint $M M_{p p} \geq 260$ $\mathrm{MeV}$ before applying the kinematic fit to events, who have

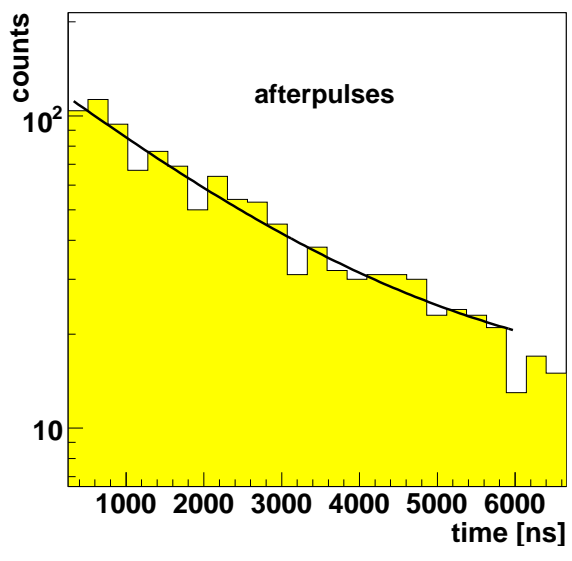

Fig. 3. Time spectrum of afterpulses detected by the delayed pulse technique installed at Quirl and Calorimeter.The logarithmic slope given by the solid curve corresponds to $\tau=$ $2.18(6) \mu \mathrm{s}$, which agrees very well with the muon lifetime. In the fit a small background of 10 counts per bin ( 1 bin corresponds to $256 \mathrm{~ns}$ ) is assumed.
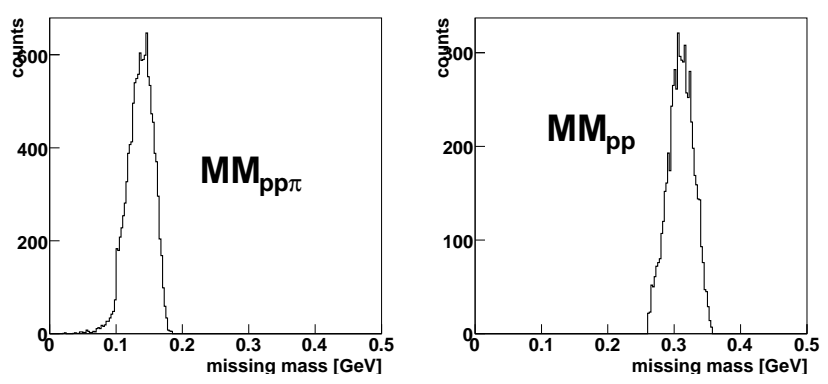

Fig. 4. Left: missing mass spectrum $M M_{p p \pi}$ of two identified protons and one identified pion with the condition $M M_{p p} \geq$ $260 \mathrm{MeV}$. Right: missing mass spectrum $M M_{p p}$ of two identified protons with the condition $100 \mathrm{MeV} \leq M M_{p p \pi} \leq 180$ $\mathrm{MeV}$.

passed both these conditions. For the kinematic fits we have one overconstraint in case of three-prong $p p \pi$ events and 3 overconstraints in case of four-prong events, where all emerging particles have been recorded in the detector.

The luminosity of the experiment was determined as $d L / d t=5 * 10^{28} \mathrm{~cm}^{-2} \mathrm{~s}^{-1}$ from the analysis of $p p$ elastic scattering, which was measured simultaneously utilizing a prescaled two-prong trigger for hits in the Ring detector. Due to their opening angle of $\delta_{p p} \approx 80^{\circ}$ between both tracks, such two-track events have both hits in the Ring. They are easily identified by using in addition the coplanarity constraint $170^{\circ}<\Delta \Phi \leq 180^{\circ}$, i.e. using the same procedure as described in more detail in Ref. [10]. Adjustment of the $p p$-elastic data in absolute height to the SAID values gives the required luminosity. All data have been efficiency corrected by MC simulations of the detector setup by using the CERN GEANT3 [13] detector simulation package, which accounts both for electromag- 
netic and hadronic interactions of the ejectiles with the detector materials.

The measurements have been conducted with a vectorpolarized proton beam providing a steady change of "spinup" and "spin-down" runs in subsequent spills of about $10 \mathrm{~s}$ duration. "Spin-up" is defined of having the spin of the protons perpendicular to the plane defined by the COSY accelerator ring and pointing to the sky (y-axis). According to the Madison convention [14] we then use a coordinate system, where the $\mathrm{z}$-axis is along the beam direction and the $\mathrm{x}$-axis points to the horizontal left corresponding to the azimuthal angle of $\Phi=0^{\circ}$ being at 9 o'clock when looking downstream the beam direction.

For the determination of the beam polarisation at the target we use again elastic scattering, determine its countrate asymmetries and compare them to the known analyzing powers of elastic scattering as compiled in the SAID database [15]. The countrate asymmetry $\epsilon(\Theta, \Phi)$ at a specific solid angle $(\Theta, \Phi)$ for a certain particle of an event is given by the difference of countrates at "spin-up" $N_{\uparrow}(\Theta, \Phi)$ and "spin-down" $N_{\downarrow}(\Theta, \Phi)$ normalized to their sum:

$$
\epsilon(\Theta, \Phi)=\frac{N_{\uparrow}(\Theta, \Phi)-N_{\downarrow}(\Theta, \Phi)}{N_{\uparrow}(\Theta, \Phi)+N_{\downarrow}(\Theta, \Phi)}=P_{y} * A_{y}(\Theta, \Phi),
$$

where $P_{y}$ is the beam polarisation and

$$
A_{y}(\Theta, \Phi)=A_{y}(\Theta) * \cos (\Phi)
$$

is the analyzing power of the reaction of interest with the particle of interest detected at the solid angle $(\Theta, \Phi)$.

In order to determine and also to monitor online the beam polarisation we have selected pp elastic scattering events with $32^{\circ} \leq \Theta_{l a b} \leq 33^{\circ}$, where the SAID database [15] gives $A_{y}=0.30$. A sample of the measured countrate asymmetry $\epsilon(\Theta, \Phi)$ is given in Fig. 5 exhibiting the expected cosine dependence on the azimuthal angle. A fit to the data with eqs.(1) and (2) yields the beam polarisation $P_{y}$. During the beamtime the beam polarisation was very stable within a few percents and with very high values of typically $P_{y}=0.87$.

Due to its cylinder symmetry the TOF detector is ideally suited for polarisation measurements. Thus, the analyzing power $A_{y}$ may be determined experimentally by use of the super ratio $R(\Theta, \Phi)$ of the four countrates for the combinations of "spin-up", spin-down" with "left" or "right" :

$$
R(\Theta, \Phi)=\sqrt{\frac{N_{\uparrow}(\Theta, \Phi) * N_{\downarrow}\left(\Theta, \Phi+180^{\circ}\right)}{N_{\downarrow}(\Theta, \Phi) * N_{\uparrow}\left(\Theta, \Phi+180^{\circ}\right)}}
$$

and

$$
A_{y}(\Theta, \Phi)=\frac{1}{P_{y}} * \frac{R(\Theta, \Phi)-1}{R(\Theta, \Phi)+1}
$$

In this superratio systematic uncertainties, detector efficiencies etc. cancel to a very large extent making thus the deduced analyzing power essentially free of systematic asymmetries, which often are not under control in "single arm" measurements with a not cylinder-symmetric detector. By using the four countrates we in addition may define an "anti-analyzing power" (instrumental asymmetry) $\bar{A}_{y}$ with the alternative super ratio $\bar{R}$ defined as

$$
\bar{R}(\Theta, \Phi)=\sqrt{\frac{N_{\uparrow}(\Theta, \Phi) * N_{\uparrow}\left(\Theta, \Phi+180^{\circ}\right)}{N_{\downarrow}(\Theta, \Phi) * N_{\downarrow}\left(\Theta, \Phi+180^{\circ}\right)}},
$$

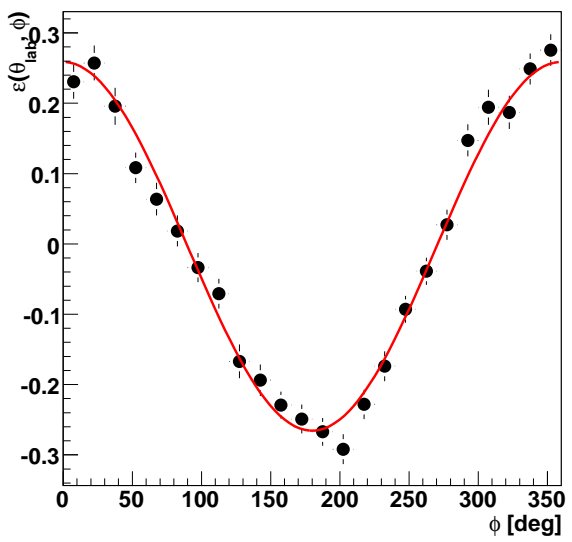

Fig. 5. Countrate asymmetry $\epsilon\left(\Theta_{l a b}, \Phi\right)$ in dependence of the azimuthal angle $\Phi$ for elastic scattering events measured in the polar angle interval $32^{\circ} \leq \Theta_{l a b} \leq 33^{\circ}$. The solid curve is a fit to the data with a $\cos (\Phi)$ dependence.

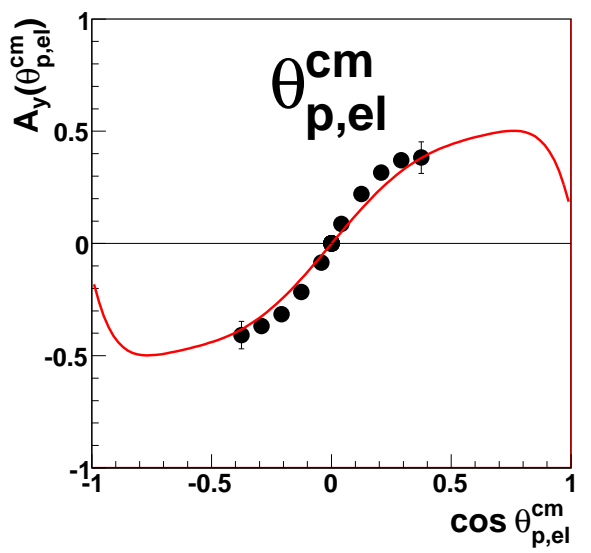

Fig. 6. Analyzing power of elastic scattering events measured in the Ring detector in comparison with the SAID database [15] (solid line).

which leads to $\overline{A_{y}}=0$ for all cases with the exception of systematic changes in beam direction when flipping the spin of the projectiles. Hence $\bar{A}_{y}$ serves as a control observable to uncover systematic asymmetries of such kind.

Due to their trivial $\cos \Phi$ dependence the analyzing powers are usually quoted as

$$
A_{y}(\Theta)=A_{y}(\Theta, \Phi=0) .
$$

In order to take into account the fully available statistics, we have sorted the data into $\Theta$ and $\Phi$ bins, calculated $A_{y}(\Theta, \Phi)$ for each of these bins and fitted for each particular $\Theta$ bin the $\Phi$ dependence of $A_{y}(\Theta, \Phi)$ by a cosine dependence. As a result we obtain for each $\Theta$ bin an $A_{y}(\Theta)$ value, which contains the full statistics of the measurements over the full $\Phi$ range.

As an example we show in Figs. 6 and 8 analyzing power and anti-analyzing power (instrumnetal asymmetry) distributions obtained for $p p$ elastic scattering events 


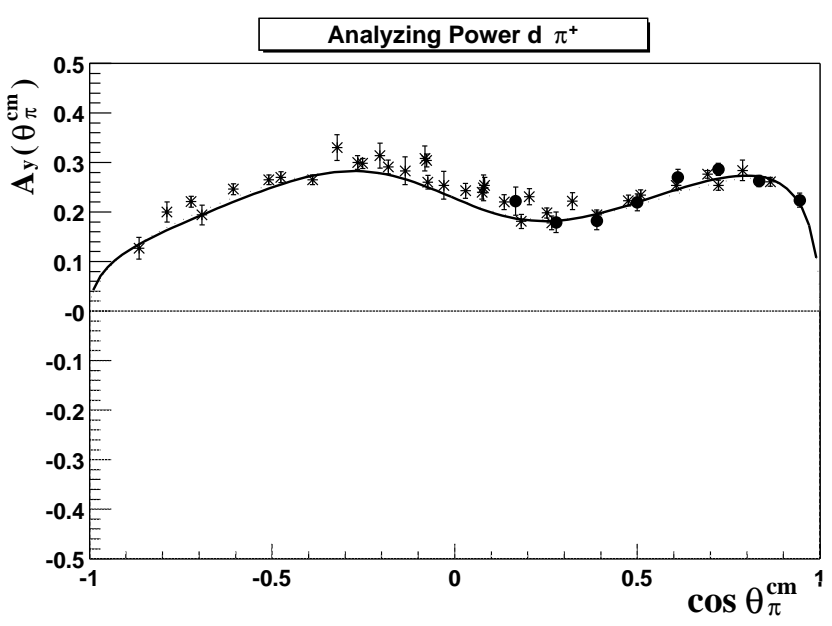

Fig. 7. Analyzing power data for the $p p \rightarrow d \pi^{+}$reaction as measured in the TOF detector (solid circles) in comparison with the SAID database [15](solid line) and LAMPF data [16] (asterisk symbols).
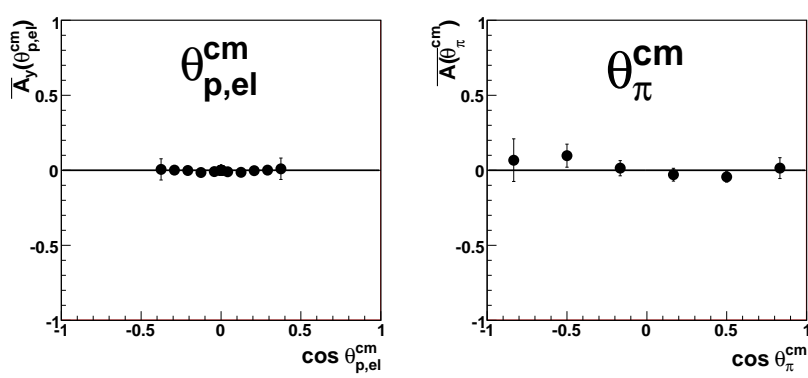

Fig. 8. "Anti-analyzing power" (instrumental asymmetry) as defined in eq.(5) for elastic scattering (left) and two-pion production events in dependence of the two-pion polar angle $\Theta_{\pi}^{c m}$ (right).

recorded in the Ring detector. The analyzing power data agree well with SAID in the measured range. As a further check of the deduced beam polarisation we have evaluated also the $p p \rightarrow d \pi^{+}$reaction utilising the same 2-prong trigger as used for the selection of elastic scattering events. The results for the selected $d \pi^{+}$events is shown in Fig. 7 in comparison with the SAID data base [15] and LAMPF data [16]. Again there is good agreement.

The anti-analyzing power (intsrumental asymmetry) data are all consistent with zero giving thus no indication of systematic asymmetries in the measurements. As a typical example of an anti-analyzing power distribution obtained from two-pion production events we show in Fig.8, right, the distribution according to the angle $\Theta_{\pi}^{c m}$ of any of the pions in the overall $\mathrm{cms}$. Again the data are consistent with zero, whereas $A_{y}\left(\Theta_{\pi}^{c m}\right) \neq 0$, see Fig. 15 .

\section{Results}

\subsection{Cross Sections - Unpolarized}

Due to the identity of the collision partners in the entrance channel the angular distributions in the overall center-ofmass system have to be symmetric about $90^{\circ}$, i.e. the full information about the reaction channels is contained already in the interval $0^{\circ} \leq \Theta^{\mathrm{cm}} \leq 90^{\circ}$. Deviations from this symmetry in the data indicate systematic uncertainties in the measurements. Hence we plot - where appropriate the full angular range, in order to show the absence of such type of systematic errors in our measurement.

Tab. 1: Total cross sections $\sigma_{\text {tot }}$ at $T_{p} \approx 750$ and 800 $\mathrm{MeV}$ for the reaction $p p \rightarrow p p \pi^{+} \pi^{-}$evaluated in this work and compared to previous measurements.

\begin{tabular}{lll}
\hline & $\sigma_{\text {tot }}[\mu \mathrm{b}]$ & \\
$T_{p}[\mathrm{MeV}]$ & this work & previous \\
\hline 750 & $1.6(2)$ & $1.6(3)^{a)}$ \\
& & $1.3(3)^{b)}$ \\
800 & $4.1(4)$ & $3(1)^{c)}$ \\
\hline a)
\end{tabular}

The evaluated total cross sections for the $p p \pi^{+} \pi^{-}$channel are given in Tab. 1 and shown in Fig. 9 together with previous results. The uncertainties assigned are based on systematics for acceptance and efficiency corrections as obtained by variation of $\mathrm{MC}$ simulations for the detector response, where we have varied the $\mathrm{MC}$ input assuming either pure phase space or some reasonable models for the reaction under consideration. For $750(800) \mathrm{MeV}$ we have collected about 1600 (6000) good events, i.e., statistical uncertainties are of minor importance for the uncertainties in the absolute total cross section.

For $T_{p}=750 \mathrm{MeV}$ total and differential cross sections for this reaction channel are very well known from previous experiments at CELSIUS [4, 18]. Hence we use the analysis of our data at this energy primarily as a check of the reliability of our measurement and data analysis. In fact, the differential distributions obtained at CELSIUS and COSY-TOF are compatible within statistical uncertainties [9, 19]. As an example we compare in Fig. 10 both data sets for the differential distributions of the invariant mass $M_{\pi \pi}$ and the opening angle $\delta_{\pi \pi}$ between the two pions in the overall cms. We also note that the values for $\sigma_{\text {tot }}$ agree very well within uncertainties.

In contrast to $T_{p}=750 \mathrm{MeV}$ there exists merely a single-arm measurement for $T_{p}=800 \mathrm{MeV}$, namely the one performed at LAMPF [17] with an estimated total cross section of $3 \mu \mathrm{b}$.

Differential cross sections for invariant masses and angular distributions obtained from our measurements are shown in Figs. 10 - 13. 


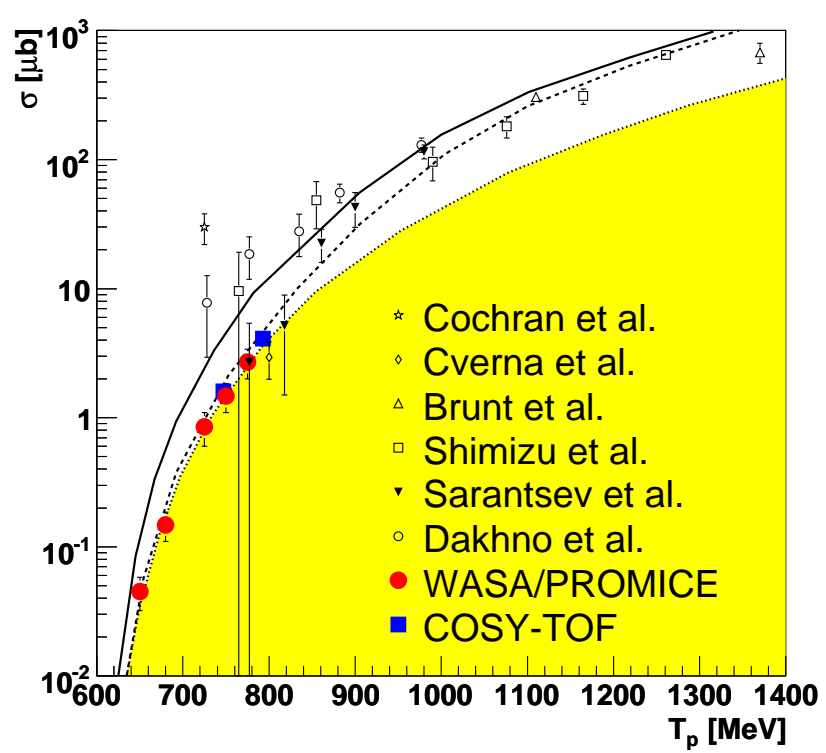

Fig. 9. Energy dependence of the total cross section. Shown are the data of this work (solid squares) together with those from CELSIUS [4,5,6] (solid circles), Gatchina - old [23] (open circles) and new [24] (solid triangles), LAMPF [17,20] (open diamond and star, respectively), KEK [22] (open squares) and Berkeley [21] (open triangles). Solid and dashed curves correspond to theoretical calculations of Ref. 3] with and without $p p$ FSI. The hatched area represents the phasespace dependence adjusted arbitrarily to the cross section at $T_{p}=750 \mathrm{MeV}$.
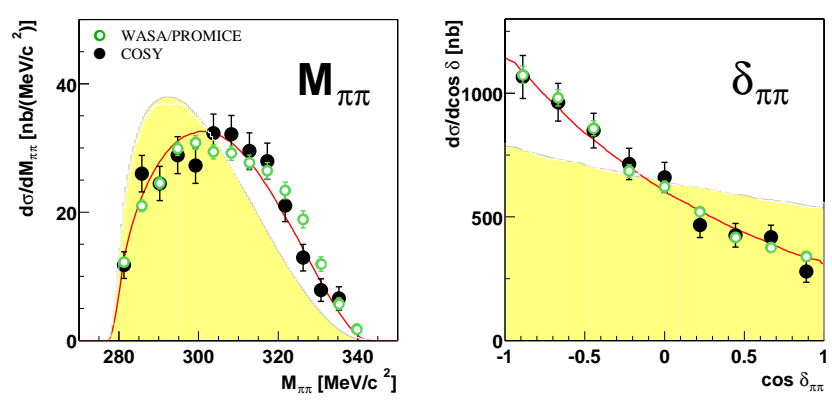

Fig. 10. Differential cross sections for the distributions of $\pi \pi$ invariant mass $M_{\pi \pi}$ (left) and $\pi \pi$ opening angle in the overall cms $\delta_{\pi \pi}$ (right) at $T_{p}=750 \mathrm{MeV}$. The data of this work (filled symbols) are compared to those obtained at CELSIUS (open symbols) as well as to phase space distributions (shaded areas) and calculations according to eq.(6) (solid lines). CELSIUS data and theoretical curves are renormalized in area $\left(\sigma_{t o t}\right)$ to the data of this work.

\subsection{Analyzing Powers}

These measurements are the first ones with polarized beam, which have been conducted for the two-pion production channel in $N N$ collisions. The angular distributions for the analyzing power have been obtained as described in detail in section 2 .
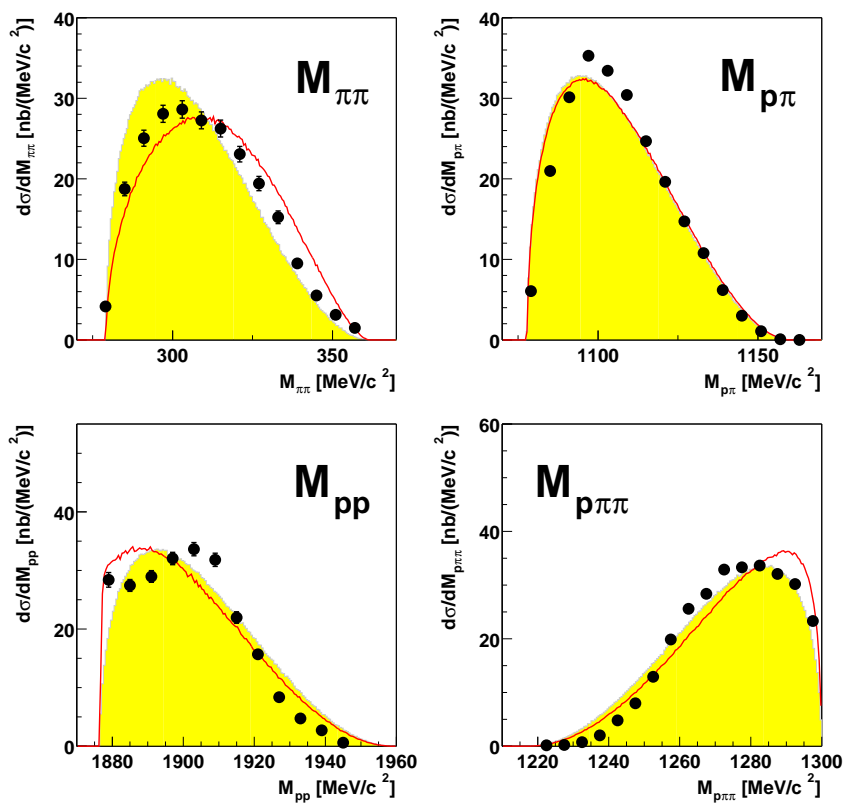

Fig. 11. Results of this work for differential distributions of the invariant masses $M_{p p}, M_{\pi \pi}, M_{p \pi}$ and $M_{p \pi \pi}$ at $T_{p}=800 \mathrm{MeV}$. They are compared to phasespace distribuions (shaded areas) as well as to calculations according to eq.(6) (solid lines).
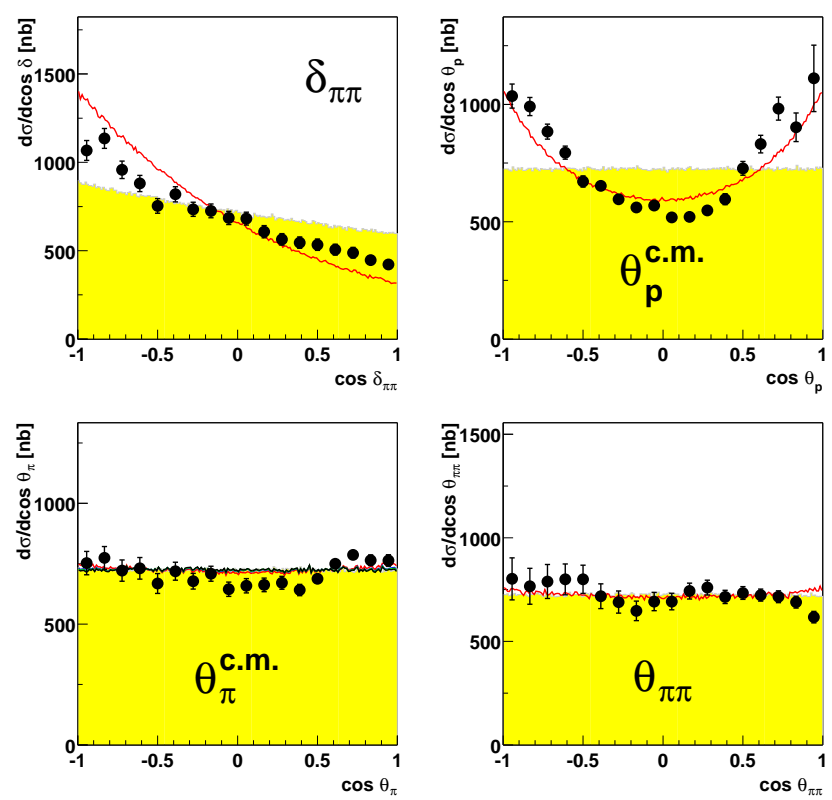

Fig. 12. The same as Fig. 11, but for of the opening angle between the two pions $\delta_{\pi \pi}$, the polar angles of protons $\Theta_{p}^{c m}$, pions $\Theta_{\pi}^{c m}$ and of the two-pion system $\Theta_{\pi \pi}^{c m}$ - all in the overall cms. 

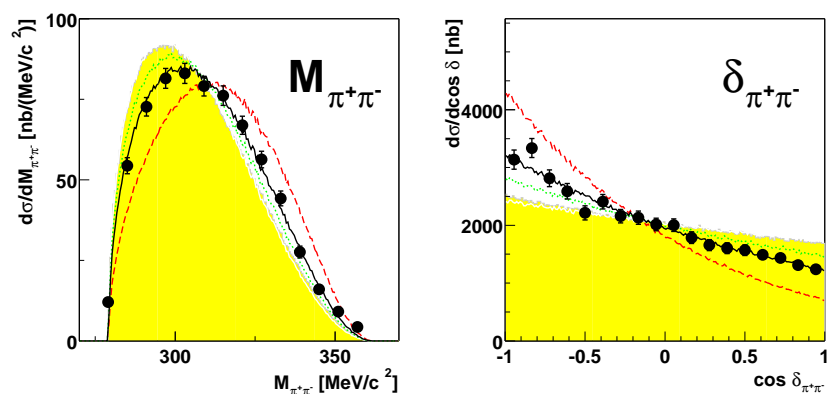

Fig. 13. Differential cross sections for the distributions of $\pi \pi$ invariant mass $M_{\pi \pi}$ (left) and $\pi \pi$ opening angle in the overall cms $\delta_{\pi \pi}$ (right) at $T_{p}=800 \mathrm{MeV}$. The data of this work are compared to phase space distributions (shaded areas) and calculations according to Ref.[3]. The dashed lines show the original calculations of Ref. 3] renormalized to the data in area, the solid lines the result, when we reduce the amplitude for the branch $N^{*} \rightarrow \Delta \pi$ by a factor of two relative to the branch $N^{*} \rightarrow N \sigma$.

For $T_{p}=750 \mathrm{MeV}$, where the statistics was quite moderate, we show in Fig. 13 our results for the $A_{y}$ distributions on the angle of any proton $\Theta_{p}^{c m}$, of any pion $\Theta_{\pi}^{c m}$, of the $\pi \pi$ system $\Theta_{\pi \pi}^{c m}$ and of any $p \pi$ system $\Theta_{p \pi}^{c m}$ - all in the overall $\mathrm{cms}$.

For $T_{p}=800 \mathrm{MeV}$, where we have much better statistics, we show in addition to these distributions (Fig. 15) also the ones, where we differentiated between $\pi^{+}$and $\pi^{-}$ particles with help of the delayed pulse technique (Fig. 16). Finally we display in Fig. 17 the analyzing powers for the angles $\Theta_{\pi}^{\pi \pi}$ of any pion in the $\pi \pi$ subsystem and for the angles $\Theta_{p}^{p p}$ of any proton in the $p p$ subsystem, both taken in the Jackson frame, i.e., having the beam axis as z-axis.

\section{Discussion of Results}

\subsection{Cross Sections - Unpolarized}

The total cross sections obtained in this work are shown in Fig. 9 together with data from previous work [17,20, 21,22, 23, 24, 4] and theoretical calculations of Ref [3]. At $T_{p}=750 \mathrm{MeV}$ our value agrees well with those obtained at CELSIUS [4,5]. At $T_{p}=800 \mathrm{MeV}$ our value is consistent with the estimate of Ref. 17] and follows also the trend given by the very recent bubble chamber results from Gatchina [24], which correct the old Gatchina results [23] for the total cross sections.

The theoretical calculations of Ref. [3] are compatible with the data from LAMPF [17, CELSIUS [4,5], Gatchina 24] and COSY-TOF for $T_{p} \leq 800 \mathrm{MeV}$ only, if set I (see Ref. [3]) for the Roper resonance parameters is used - and if the $p p$ final state interaction (FSI) is neglected (lower dashed curve in Fig. 9). Taking into account the FSI (solid curves) the theoretical calculations overestimate the cross sections in the near-to-threshold region by as much as an order of magnitude.
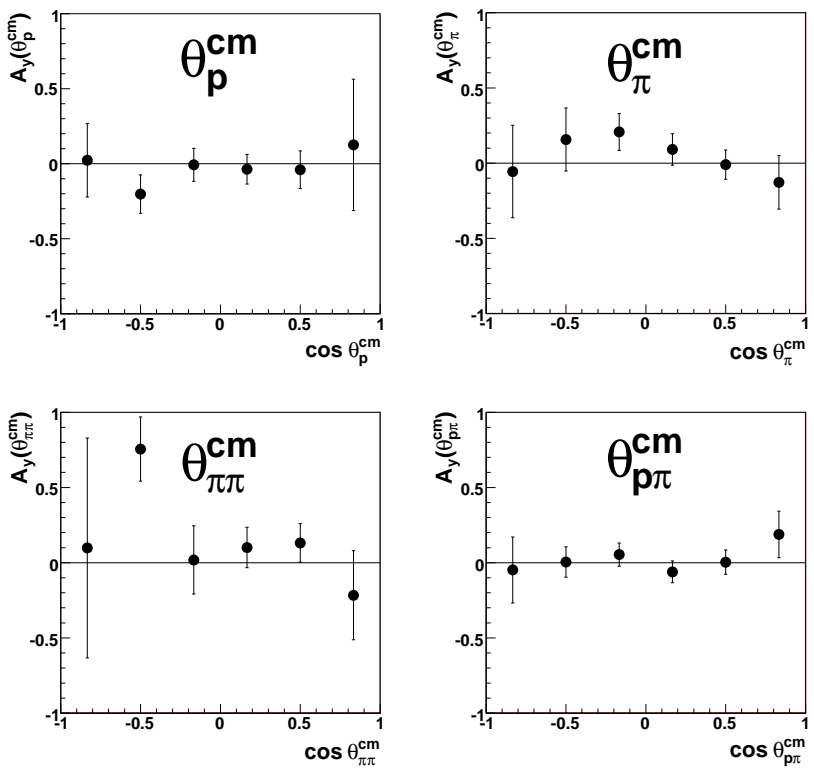

Fig. 14. Analyzing power distributions for the polar angles $\Theta_{p}^{c m}, \Theta_{\pi}^{c m}, \Theta_{\pi \pi}^{c m}$ and $\Theta_{p \pi}^{c m}$ at $T_{p}=750 \mathrm{MeV}$.
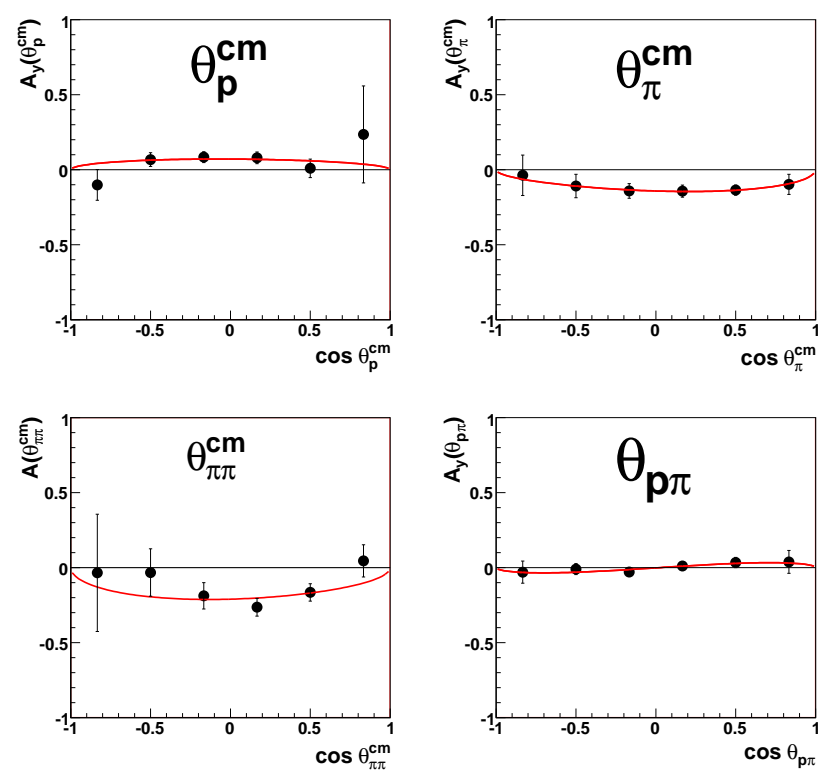

Fig. 15. Analyzing power distributions for the polar angles $\Theta_{p}^{c m}, \Theta_{\pi}^{c m}, \Theta_{\pi \pi}^{c m}$ and $\Theta_{p \pi}^{c m}$ at $T_{p}=800 \mathrm{MeV}$. The solid curves show a fit according to eq.(7).

For the discussion in particular of the data taken at $T_{p}=800 \mathrm{MeV}$, we compare our data in Figs. $10-13$ with phase phase distributions denoted by the hatched areas in the figures, with theoretical calculations of Ref. [3] (Fig. 13 only) as well as with simplified calculations of Roper excitation and decay according to the ansatz for the Roper amplitude

$$
A \sim 1+c \mathbf{k}_{\mathbf{1}} * \mathbf{k}_{\mathbf{2}}\left(\mathbf{3} \mathbf{D}_{\boldsymbol{\Delta}^{++}}+\mathbf{D}_{\Delta^{\mathbf{0}}}\right),
$$



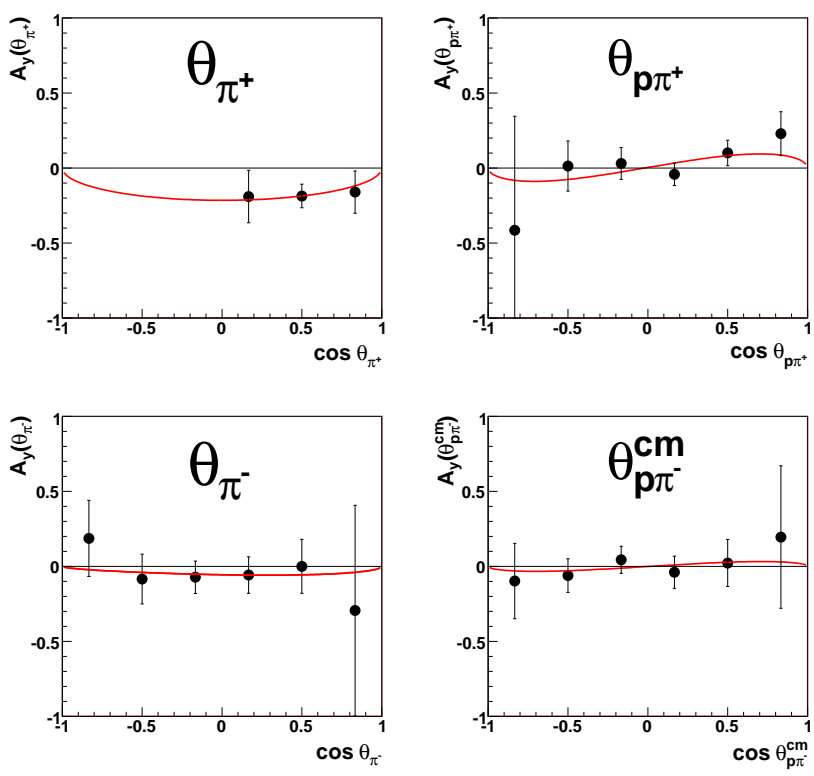

Fig. 16. Analyzing power distributions for the polar angles $\Theta_{\pi^{+}}^{c m}, \Theta_{\pi^{-}}^{c m}, \Theta_{p \pi^{+}}^{c m}$ and $\Theta_{p \pi^{-}}^{c m}$ at $T_{p}=800 \mathrm{MeV}$, i.e. separated for positively and negatively charged pions. The curves show a fit according to eq.(7).
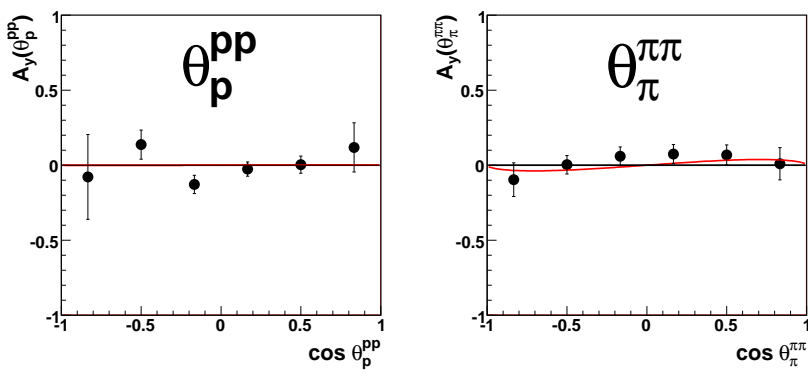

Fig. 17. Analyzing power distributions for the polar angles $\Theta_{p}^{p p}, \Theta_{\pi}^{\pi \pi}$ in the $p p$ and $\pi \pi$ subsystem (Jackson frame) at $T_{p}=$ $800 \mathrm{MeV}$. The curves show a fit according to eqs.(7) and (8), respectively.

which multiplies the expressions for $\sigma$ exchange, FSI and $N^{*}$ propagator (see Refs. [4,5]) and where $D_{\Delta}$ denotes the $\Delta$ propagator. The first term in eq. (6) stands for the Roper decay into the $N \sigma$ channel. The second term gives the Roper decay via the $\Delta$ resonance, i.e. $N^{*} \rightarrow \Delta \pi \rightarrow$ $N \pi \pi$, where the scalar product of the pion momenta $\mathbf{k}_{\mathbf{1}}$ and $\mathbf{k}_{\mathbf{2}}$ stands for the double p-wave emission of the pions in this process. Whereas the first term provides a phasespace like behavior of the observables, the second term is proportional to the cosine of the $\pi \pi$ opening angle in the cms and hence provides both a change in the slope of the $\delta_{\pi \pi}$ spectrum and a shift of the $M_{\pi \pi}$ distribution towards higher masses. From this apparent signature in these observables the parameter c can be fixed easily by corresponding data.
The coefficient $\mathrm{c}$ contains the branching of the decays $N^{*} \rightarrow N \sigma$ and $N^{*} \rightarrow \Delta \pi$. Adjustment of $\mathrm{c}$ to the CELSIUS data at $T_{p}=750$ and $775 \mathrm{MeV}$ [5] resulted in a branching ratio of $R=\Gamma_{N^{*} \rightarrow \Delta \pi \rightarrow N \pi \pi} / \Gamma_{N^{*} \rightarrow N \sigma}$ between the two-pion decays of the Roper resonance of $R=3(1)$, if the pole of the Roper resonance is assumed to be at its nominal value of $1440 \mathrm{MeV}$ - in good agreement with the PDG value of 4(2) 25, but more precise. However, if the pole is taken to be at $1371 \mathrm{MeV}$, which meanwhile is considered to be more correct [25, 26, 27, 28, 29, then this ratio changes to $R=1.0$ (1). Calculations with this value of $c$ are shown in Figs. $10-12$ by the solid lines. They give a qualitative description of the main features in the differential data. At a closer look, however, they provide a shift in the $M_{\pi \pi}$ spectrum and a slope in the $\delta_{\pi \pi}$ spectrum, which is somewhat too large. This suggests that the $N^{*} \rightarrow \Delta \pi$ decay is even weaker than the $N^{*} \rightarrow N \sigma$ decay - as was also obtained in a very recent analysis of photonand pion-induced reactions [29]. The result obtained here is also in agreement with a recent analysis of $p p \rightarrow p p \pi^{0} \pi^{0}$ data taken at CELSIUS-WASA [33.

Fig. 13 shows the full calculations of Ref.[3], which also include reaction processes other than just the Roper excitation. The dotted line corresponds to calculations with the same branching for the $N^{*} \rightarrow N \sigma$ and $N^{*} \rightarrow \Delta \pi$ decay processes as used with the simplified ansatz (6). We see by comparison of Figs. 11 and 12 with Fig. 13 that full and simplified calculations give nearly the same result, which means that the terms neglected in the ansatz (6) are of minor importance. In fact, if we now reduce the $N^{*} \rightarrow \Delta \pi$ amplitude by a factor of two in the calcultions of Ref.[3] - as done in Ref. [33] - then we get full agreement with the data.

\subsection{Analyzing Powers}

The analyzing powers taken at $T_{p}=750 \mathrm{MeV}$ shown in Fig. 14 are essentially compatible with zero within their large statistical uncertainties. Because of that we do not discuss them here in more detail and concentrate on the data at $T_{p}=800 \mathrm{MeV}$, which have much better statistics. We only note in passing, that the trend in the analyzing powers at $T_{p}=750 \mathrm{MeV}$ is just opposite in sign to that in the data at $T_{p}=800 \mathrm{MeV}$. The reason for it is not known to us.

At $T_{p}=800 \mathrm{MeV}$ the data displayed in Figs. 15 17 exhibit significant analyzing powers with values up to $A_{y}=0.3$. Whereas the analyzing powers in the $p p$ and $\pi \pi$ subsystems are very small and compatible with zero, they are quite substantial in the overall cms for $\Theta_{\pi}^{c m}$ and $\Theta_{\pi \pi}^{c m}$. The analyzing power of the protons in the overall $\mathrm{cms}$, i.e. $A_{y}\left(\Theta_{p}^{c m}\right)$, is again small.

The analyzing powers for $\Theta_{\pi}^{c m}$ and $\Theta_{\pi \pi}^{c m}$ are of course not decoupled. Since for the latter the analyzing power is largest, the source for polarization producing effects is expected to be connected with this observable.

From general considerations [30,31] of meson production in $N N$ collisons the analyzing powers in the overall 
cms has the following $\Theta$ dependences (see appendix for more details):

$$
A_{y}=a \sin (\Theta)+b \sin (2 \Theta),
$$

whereas in the $p p$ subsystem we have only a $2 \Theta$ dependence:

$$
A_{y}=b^{\prime} \sin (2 \Theta) \text {. }
$$

A more detailed description within the partial wave concept is given in the appendix. The solid lines in Figs. 15 17 show fits to the data with eqs. (7) and (8), respectively. As one can see from Figs. $15-17$, the coefficients $a$ and $b$ are only significantly different from zero for $\Theta_{p}^{c m}, \Theta_{\pi}^{c m}$ and $\Theta_{\pi \pi}^{c m}$ distributions. In addition the latter ones are essentially described by a $\sin (\Theta)$ dependence with the $\sin (2 \Theta)$ dependence being of minor importance or negligible.

Finally we look into possible differences between $\pi^{+}$ and $\pi^{-}$angular distributions by exploiting the delayed pulse technique to identify the $\pi^{+}$particle in an event. That way we obtain the data distinguished between $\pi^{+}$ and $\pi^{-}$observables in Fig. 16. Though the statistics gets worse due to the finite $\pi^{+}$identification efficiency, we find the analyzing powers for the $\pi^{+}$observables in $\Theta_{\pi^{+}}^{c m}$ and $\Theta_{p \pi^{+}}^{c m}$ to be systematically larger than for those for the corresponding $\pi^{-}$observables. Such a difference indicates a significant contribution of isovector $\pi \pi$ pairs.

From this and the requested sine dependence of the analyzing power we learn that the driving term for finite analyzing powers must be connected primarily with the $\pi^{+}$momentum and be of the form

$$
\sigma \cdot\left(\mathbf{q} \times \mathbf{k}_{\pi^{+}}\right),
$$

where $\mathbf{q}$ and $\mathbf{k}_{\pi^{+}}$are momentum transfer between the nucleons and the $\pi^{+}$momentum, respectively, and $\sigma$ is the Pauli spin operator. Since the momentum transfer close to threshold is approximately given by the initial momentum in the overall $\mathrm{cms}$, we arrive with such an operator at the observed $\sin \left(\Theta_{\pi^{+}}^{c m}\right)$ dependence.

Since to lowest order spinflip terms like (9) are connected with $\mathrm{p}$-waves, whereas nonspinflip terms are connected with s-waves, the analyzing power arises to lowest order from an interference of $\mathrm{S}$ - and $\mathrm{P}$-waves in the entrance channel. The dominating reaction process at $T_{p} \approx$ $800 \mathrm{MeV}$ is via the Roper excitation and hence fed by the ${ }^{1} S_{0}$ partial wave in the entrance channel. Processes involving the operator of eq. (9) evolve via the ${ }^{3} P_{1}$ partial wave. From inspection of the formulas given in Ref.[3] we see that neither Roper nor $\Delta \Delta$ excitation and decays provide such an operator. The only process, which provides such an operator in the theoretical investigations of Ref. [3], is single $\Delta$ excitation and decay in combination with pion rescattering on the other vertex as given by the graphs (10) and (11), Fig.1 of Ref. [3], or graph (9), which gives a successive excitation of a $\Delta$ on a single nucleon. However, in these calculations such graphs give only tiny contributions to the cross section. On the other hand the analyzing power is an ideal tool to reveal such small amplitudes by their interference with large amplitudes. Hence it appears very interesting to check by realistic calculations, whether such graphs could be the origin of the finite analyzing powers we observe. As pointed out above, the calculations of Ref. [3] are done in the plane wave limit, i.e., are not valid for polarization observables. Hence an improved calculation, which includes initial state interactions, would be of great help to understand the polarization data on a quantitative basis.

Another interesting aspect could be the consideration of a possible influence of the $\Delta(1600)$ resonance. Though its resonance energy is as high as that of the $\Delta \Delta$ excitation, its unusually large width of $\approx 350 \mathrm{MeV}[25$ is in favor of taking part in the two-pion production already close to threshold. Indeed, as has been noted already in Ref. [32], the isotensor two-pion production amplitude is observed to be unusually large. And the $\Delta(1600)$ resonance is the only resonance candidate to contribute to the isotensor amplitude near threshold in addition to the $\Delta \Delta$ process.

The analyzing power arises from the interference of spinflip and non-spinflip terms in the reaction amplitude, whereas the unpolarized cross section is just the sum of their moduli squared. If we denote the non-spinflip term by $g$ and the strength of the spinflip term which multiplies the operator (9) by $\mathrm{h}$, then we have

$$
\sigma A_{y}=-2 \operatorname{Im}\left(g^{*} h\right) k_{\pi^{+}} \sin \left(\Theta_{\pi^{+}}^{c m}\right)
$$

and

$$
\sigma=|g|^{2}+|h|^{2} k_{\pi^{+}}^{2} \sin ^{2}\left(\Theta_{\pi^{+}}^{c m}\right) \leq|g|^{2}\left(1+A_{y}^{2} / 4\right),
$$

i.e., a $30 \%$ effect in the analyzing power produces only a $2 \%$ effect of non-isotropy in the differential cross section. Such an effect is not observable in the cross section with the statistics accumulated in this measurement.

\section{Summary}

The experimental results presented in this work constitute the first exclusive measurements of $\pi^{+} \pi^{-}$production in $N N$ collisions at $T_{p}=800 \mathrm{MeV}$ and provide the first polarization data at all for this production process. The unpolarized differential cross sections support the findings of previous analyses that Roper excitation and decay is the dominant two-pion production process. The data favor the direct decay of the Roper resonance into the $N \sigma$ channel over its decay via the $\Delta$ resonance. This in turn supports the breathing mode nature of the Roper resonance.

The observation of sizable analyzing powers in combination with the fact that neither Roper nor $\Delta \Delta$ processes contribute to substantial analyzing powers, point to a small amplitude of different nature, which interferes with the strong Roper amplitude. Candidates for such a small amplitude could be $\Delta$ excitation processes, where on the one nucleon vertex a $\Delta(1232)$ or $\Delta(1600)$ is excited followed by its transition $\Delta \rightarrow \Delta \pi \rightarrow N \pi \pi$, which would be the first time that such a process has been sensed in $N N$ collisions.

This work has been supported by BMBF, DFG (Europ. Graduiertenkolleg 683) and COSY-FFE. We acknowledge valuable discussions with Eulogio Oset, Murat Kaskulov and Alexander 


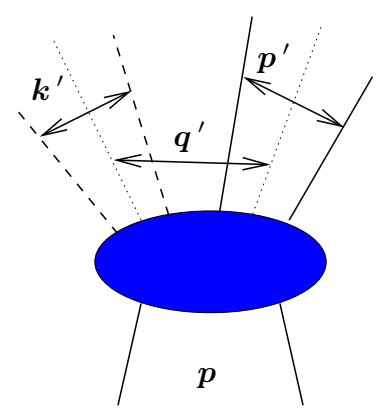

Fig. 18. Definition of the coordinate system. Dashed (solid) lines denote pions (nucleons).

Sibirtsev. We are particularily indebted to Luis Alvarez-Ruso, who made his computer code available to us.

\section{Appendix}

In this appendix the general structure of the amplitudes is derived. Since none of the observables shows a stricing energy dependence and the energy is still not too far above threshold it appears justified to work with the lowest partial waves only. Hence we will only derive the angular structures of differential cross section and analyzing power up to order $Q^{2}$, were $Q$ is any of the momenta in the final state as defined in Fig. 18 .

Before we can identify the partial waves that are relevant given the assumption formulated above, we need to briefly comment on the relevant selection rules. The Pauli principle enforces that a proton pair of spin 0 (1) has even (odd) parity. As a consequence, for unpolarized observables $p p S$-waves and $p p P$-waves never interfere. Since in the experiment presented the polarization of the outgoing protons is not measured, no interferences of $p p P$ - and $S$-waves in the final state are possible. We therefore only need to consider $p p P$-waves in the final state in combination with $s$-waves for the other two subsystems ( $\pi \pi$ and $\pi \pi$ relative to $p p$ ).

There are 4 partial waves possible with an NN p-wave in the final state $\left({ }^{3} P_{j} \rightarrow{ }^{3} P_{j} s[s]\right.$ with $j=0,1,2$ and ${ }^{3} F_{2} \rightarrow{ }^{3} P_{2} s[s]$, where we used the notation ${ }^{2 S+1} L_{j} \rightarrow$ ${ }^{2 S^{\prime}+1} L_{j^{\prime}}^{\prime} \bar{l}[l]$, with $S, L, j\left(S^{\prime}, L^{\prime}, j\right)$ for spin, angular momentum, and total angular momentum of the $p p$ pair in the initial (final) state, $l$ for the angular momentum of the pion pair, and $\bar{l}$ for the angular momentum of the pion pair relative to the $p p$ pair. It is straight forward to see that only 3 independent operator structures are possible and we define

$\mathcal{M}^{P}=d_{1}(\boldsymbol{S} \cdot \hat{p})\left(\boldsymbol{S}^{\prime} \cdot \boldsymbol{p}^{\prime}\right)+d_{2}\left(\boldsymbol{S} \cdot \hat{p}^{\prime}\right)\left(\boldsymbol{S}^{\prime} \cdot \boldsymbol{p}\right)+d_{3}\left(\boldsymbol{S} \cdot \boldsymbol{S}^{\prime}\right)\left(\hat{p} \cdot \boldsymbol{p}^{\prime}\right)$.

The partial wave amplitudes are linear combinations of the $d_{i}$.

Terms of at most quadratic dependence on the final momenta in combination with $p p S$-waves in the final state can emerge from overall $S$-waves interfering either with themselves, $p$-waves in the subsystems or $d$-waves in the subsystems. We therefore need to consider in total 6 partial waves for this part that we parametrize as

$$
\begin{aligned}
\mathcal{M}^{S}=a & +b_{1}(\boldsymbol{S} \times \hat{p}) \cdot \boldsymbol{q}^{\prime}+b_{2}\left(\left(\boldsymbol{q}^{\prime} \cdot \hat{p}\right)^{2}-\frac{1}{3} \boldsymbol{q}^{\prime 2}\right)+ \\
& +c_{1}(\boldsymbol{S} \times \hat{p}) \cdot \boldsymbol{k}^{\prime}+c_{2}\left(\boldsymbol{q}^{\prime} \cdot \boldsymbol{k}^{\prime}\right)+ \\
& +c_{3}\left(\left(\hat{p} \cdot \boldsymbol{q}^{\prime}\right)\left(\hat{p} \cdot \boldsymbol{k}^{\prime}\right)-\frac{1}{3}\left(\boldsymbol{q}^{\prime} \cdot \boldsymbol{k}^{\prime}\right)\right),
\end{aligned}
$$

where the $a$ term corresponds to ${ }^{1} S_{0} \rightarrow{ }^{1} S_{0} s[s]$, the $b_{1}$ term to ${ }^{3} P_{1} \rightarrow{ }^{1} S_{0} p[s]$, the $b_{2}$ term to ${ }^{1} D_{2} \rightarrow{ }^{1} S_{0} d[s]$, the $c_{1}$ term to ${ }^{3} P_{1} \rightarrow{ }^{1} S_{0} s[p]$, the $c_{2}$ term to ${ }^{1} S_{0} \rightarrow{ }^{1} S_{0} p[p]$, and the $c_{3}$ term to ${ }^{1} D_{2} \rightarrow{ }^{1} S_{0} p[p]$. The full matrix element is given by

$$
\mathcal{M}=\mathcal{M}^{S}+\mathcal{M}^{P} .
$$

Using the formulas of chapter 4.3 of Ref. [31 it is straightforward to calculate both unpolarized cross sections as well as analyzing powers from the expressions given above. We find (dropping terms more than quadratic with respect to the final momenta)

$$
\begin{aligned}
4 \sigma_{0}=|a|^{2}+ & \left|b_{1}\right|^{2} q^{\prime 2} \sin \left(\theta_{q^{\prime}}\right)^{2}+\left|c_{1}\right|^{2} k^{\prime 2} \sin \left(\theta_{k^{\prime}}\right)^{2}+ \\
+ & 2 \operatorname{Re}\left\{a^{*} b_{2} q^{2}\left(\cos \left(\theta_{q^{\prime}}\right)^{2}-\frac{1}{3}\right)+\right. \\
+ & \frac{2}{3} a^{*} c_{3} q^{\prime} k^{\prime} \cos \left(\theta_{q^{\prime}}\right) \cos \left(\theta_{k^{\prime}}\right)+ \\
+ & \left(a^{*} c_{2}-\frac{1}{3} a^{*} c_{3}+b_{1}^{*} c_{1}\right) k^{\prime} q^{\prime} \\
& \left.\sin \left(\theta_{k^{\prime}}\right) \sin \left(\theta_{q^{\prime}}\right) \cos \left(\phi_{q^{\prime}}-\phi_{k^{\prime}}\right)\right\} \\
+ & p^{\prime 2}\left(\left|d_{1}\right|^{2}+\left|d_{2}\right|^{2}\right)+ \\
+ & p^{\prime 2} \cos \left(\theta_{p^{\prime}}\right)^{2}\left(3\left|d_{3}\right|^{2}+2 \operatorname{Re}\left\{d_{1}^{*} d_{2}+d_{1}^{*} d_{3}+d_{2}^{*} d_{3}\right\}\right) \\
4 \sigma_{0} A_{y}=- & \operatorname{Im}\left\{\left(d_{1}^{*} d_{2}+d_{1}^{*} d_{3}-d_{2}^{*} d_{3}\right) p^{\prime 2} \sin \left(2 \theta_{p^{\prime}}\right) \cos \left(\phi_{p^{\prime}}\right)\right. \\
& \left.+2 b_{1}^{*} a q^{\prime} \sin \left(\theta_{q^{\prime}}\right) \cos \left(\phi_{q^{\prime}}\right)+2 c_{1}^{*} a k^{\prime} \sin \left(\theta_{k^{\prime}}\right) \cos \left(\phi_{k^{\prime}}\right)\right\} .
\end{aligned}
$$

Note that, as a consequence of the fact that two amplitudes with a spin singlet entrance channel can not interfere in the analyzing power, the expression for the polarization observable has a much simpler structure.

\section{References}

1. T. E. O. Ericson and W. Weise, "Pions and Nuclei," Clarendon Press Oxford (1988)

2. C. Hanhart, J. Haidenbauer, O. Krehl and J. Speth, Phys. Lett. B 444 (1998) 25.

3. L. Alvarez-Ruso, E. Oset, E. Hernandez, Nucl. Phys. A633, 519 (1998) and priv. comm.

4. W. Brodowski et al., Phys.Rev. Lett.88, 192301 (2002)

5. J. Pätzold et al., Phys. Rev. C67, 052202(R) (2003)

6. J. Johanson et al., Nucl. Phys. A712,75 (2002)

7. A. Hassan et al., Nucl. Inst. Meth. A425, 403 (1999)

8. M. Dahmen et al., Nucl. Inst. Meth. A348, 97 (1994)

9. J. Kress, PhD thesis Univ. Tübingen 2003,

http://w210.ub.uni-tuebingen.de/dbt/volltexte/2003/939

10. S. Abd El-Samad et al., Eur. Phys. J. A30, 443 (2006) 
11. H. Calen et al., Nucl. Inst. Meth. A379, 57 (1996)

12. J. Zabierowski et al., Phys. Scripta T99, 159 (2002)

13. CERN Computing and Networks Divison, GEANT - Detector description and Simulation Tool, CERN Program Library

14. H. H. Barshall and W. Haeberli, Proc. Int. Conf. on Polarization Phenomena in Nuclear Reactions, University of Wisconsin Press, Madison 1971

15. R. A. Arndt et al., Phys. Rev. C62, 034005 (2000); SAID data base see also http://said.phys.vt.edu

16. W. B. Tippens et al., Phys. Rev. C36, 1413 (1987)

17. F. H. Cverna et al., Phys. Rev. C23, 1698 (1981)

18. J. Pätzold, PhD thesis Univ. Tübingen 2002, http://w210.ub.uni-tuebingen.de/dbt/volltexte/2002/550

19. A. Erhardt, PhD thesis Univ. Tübingen, to be published

20. D. R. F.Cochran et al., Phys. Rev. D6, 3085 (1972)

21. C. D. Brunt et al., Phys. Rev. 187, 1856 (1969)

22. F. Shimizu et al., Nucl. Phys. A386, 571 (1982)

23. L. G. Dakhno et al., Sov.J. Nucl. Phys. 37, 540 (1983)

24. V. V. Sarantsev et al., submitted to Phys. Atomic Nucl. Phys. - Yad. Fiz. and priv. comm.

25. Particle Data Group, J. Phys. G33, 1 (2006)

26. R. A. Arndt et al., Phys. Rev. C74, 045205 (2006)

27. M. Ablikim et al., Phys.Rev. Lett.97, 062001 (2006)

28. H. P. Morsch and P. Zupranski, Phys.Rev. C61, 024002 (2002) and references therein

29. A. V. Sarantsev et al.,Phys. Lett. B659, 94 (2008); arXiv:0707.359 [hep-ph]

30. H. O. Meyer et al., Phys. Rev. C63, 064002 (2001) and references therein

31. C. Hanhart, Phys. Rep. 397, 155 (2004)

32. J. Johanson et al., Nucl. Phys. A712, 75 (2002)

33. T. Skorodko et al., Eur. J. Phys. A35, 317 (2008) 